\title{
Well Being in Neurologic Illness
}

\author{
Michael Staton ${ }^{1}$, Lizzy Freeman ${ }^{1}$, Mona Stecker ${ }^{1,2 *}$ and Mark Stecker ${ }^{1,3}$ \\ ${ }^{1}$ Department of Neuroscience, Joan C. Edwards School of Medicine, Huntington WV 25705, USA \\ ${ }^{2}$ Department of Nursing, Winthrop University Hospital, Mineola NY 11501, USA \\ ${ }^{3}$ Department of Neuroscience, Winthrop University Hospital, Mineola NY 11501, USA
}

\begin{abstract}
Patients with neurological illness often have both diminished Quality Of Life (QOL) and diminished feelings of Well Being (WB) because of both the underlying disease, and the adverse effects of medications, especially Antiepileptic Drugs (AED's). The purpose of this pilot study is to evaluate whether a simple index of WB based upon the World Health Organization survey on WB (WHO-5) can provide useful information about patients with epilepsy.

Data was obtained from WB surveys of 629 patients presenting to a neurology/epilepsy clinic. This data is used to explore the relationship between WB and neurologic illness. In particular the effects of epilepsy, AED use, age and the effect of serial visits to the provider were studied.

Although the patients with epilepsy were younger than the other patients in the study, a factorial ANOVA suggested that there were statistically significant reductions in WB that could be attributed to both epilepsy and increased age. Further analysis focused on individual questions in the survey revealed the largest difference attributable to epilepsy centered around an investigator formulated question relating to being able to complete all activities of daily life. This is expected because epilepsy restricts important functions such as driving. AED use had no significant effect on WB. However, patients with epilepsy have improved WB scores with serial visits to the clinic while the other patients did not.
\end{abstract}

This pilot study demonstrates that a very simple marker of well-being is powerful enough to elucidate complex effects of many variables in a population of patients presenting to an epilepsy clinic.

Keywords: Epilepsy; Well being; Quality of life; Neurology clinic; WHO-5; Antiepileptic drugs

\section{Introduction}

As many neurologic illnesses are controlled but not cured by medications, it is important to optimize both the patient's physical symptoms and their overall quality of life (QOL). This is especially true because of the high psychiatric co-morbidities in patients with neurologic illness $[1,2]$. This is exemplified in the case of epilepsy where it is important to balance control of its objective manifestations, such as seizure frequency and seizure severity, against the adverse effects that anti-epileptic drugs (AED) have on QOL [2]. These adverse effects are thought to include not only physical side effects such as ataxia, nausea, etc. but also psychiatric changes such as depression and an increased risk for suicide [3]. This accentuates the need measure and monitor QOL $[1,4]$ in neurologic patients.

Quality of life is a broad concept that often refers to a patients overall health including both mental and physical health, personal beliefs, and social relationships [5]. One component of QOL is the sense of personal well being (WB) which is the evaluation of one's own condition in life [6]. The overall purpose of this paper is determine whether a simple survey of WB based upon the existing World Health Organization (WHO-5) well being index [7] can be helpful in understanding the factors that modulate the effects of neurologic illness, on patients' lives. The first specific goal of this experiment was to compare WB in patients with and without epilepsy and to determine whether anti-epileptic drugs (AED's) had any effect on WB. The second goal was to examine the effects that age and serial visits to a neurology practitioner had on WB.

\section{Methods}

\section{Subjects and environment}

As part of the standard clinical assessments of patients of 2 neurology providers, each patient arriving in the office was asked to fill out a 10-item survey about their well being (WB) (Figure 1) which the provider was able to review during the patient visit. Permission from the Marshall University IRB (193739-2) was obtained to retrospectively analyze this data. All data reviewed was from patient visits between $1 / 2009$ and 5/2011. During this period, a total of 629 patients (from 835 office visits) completely filled out the survey. The mean age of the patients was $45.3(\mathrm{SD}=17)$. Epilepsy was the primary diagnosis in $23.5 \%$ of the patients, in $14.5 \%$ it was unclear whether the patient did or did not have epilepsy and $62.0 \%$ did not have an epilepsy diagnosis. This included patients with multiple medical and psychiatric conditions. $12 \%$ of the patients without epilepsy had nonepileptic events, $12 \%$ had migraine, $12 \%$ had movement disorders, $5 \%$ had chronic pain syndromes, $4 \%$ ataxia, $4 \%$ stroke, $4 \%$ multiple sclerosis. There were multiple other diagnoses were encountered with diminishing frequency. In the patients with epilepsy, $42.6 \%$ were on polytherapy. The 113 patients not treated with polytherapy were on none or 1 of 10 different medications (Table 1).

\section{Materials}

The tool used to asses WB was a survey composed of 5 questions from the World Health Organization WHO-5 well being index [7],

${ }^{*}$ Corresponding author: Mark M. Stecker, Department of Neuroscience, Winthrop University Hospital, 7 Saint James Street North, Garden City, NY, USA, Tel: +1 (516) 663-4771; Fax: +1 (516) 663-8246; E-mail: mmstecker@gmail.com

Received May 07, 2012; Accepted May 09, 2012; Published May 13, 2012

Citation: Staton M, Freeman L, Stecker M, Stecker M (2012) Well Being in Neurologic Illness. J Neurol Neurophysiol 3:131. doi:10.4172/2155-9562.1000131

Copyright: ( 2012 Staton M, et al. This is an open-access article distributed under the terms of the Creative Commons Attribution License, which permits unrestricted use, distribution, and reproduction in any medium, provided the original author and source are credited. 
Citation: Staton M, Freeman L, Stecker M, Stecker M (2012) Well Being in Neurologic Illness. J Neurol Neurophysiol 3:131. doi:10.4172/21559562.1000131

\begin{tabular}{|c|c|}
\hline Medication & \\
\hline None & $18.5 \%$ \\
\hline Clonopin & $0.9 \%$ \\
\hline Depakote & $11.5 \%$ \\
\hline Dilantin & $22.1 \%$ \\
\hline Keppra & $11.5 \%$ \\
\hline Lamictal & $12.4 \%$ \\
\hline Neurontin & $2.7 \%$ \\
\hline Phenobarbital & $1.8 \%$ \\
\hline Tegretol & $10.6 \%$ \\
\hline Topamax & $1.8 \%$ \\
\hline Trileptal & $6.2 \%$ \\
\hline
\end{tabular}

${ }^{\mathrm{a} A E D}=$ Antiepileptic Drugs

Table 1: Percentage of patients on each AEDa within the monotherapy - epilepsy group.

which has been extensively validated $[8,9]$. In addition, 5 additional questions constructed by the investigators to inquire about other aspects of the patient's WB were also part of the survey (Figure 1). The response to all questions is on a 6 point Likert scale ranging from 0 -("at no time", poor quality) to 5-("all of the time", good quality). The total well being index is the sum of the results from all 10 questions which ranged between 0 (poor WB) and 50 (highest WB).

\section{Data analysis}

The reliability of the WB data in this patient population was checked by using both Spearman rank correlation and linear regression analysis to determine whether the scores from the WHO-5 questions [7] were correlated with those additional 5 questions added at the beginning of the survey by the investigators. Strong positive correlations between the two sets of questions suggest that that the questions are measuring similar aspects of WB and help confirm validity in this patient population

Using the total WB scores, Student's t-test was applied to determine whether there was a difference in WB in patients with definite epilepsy and those without it, excluding patients in which the diagnosis was unclear. A similar t-test was used to determine whether the age of patients with epilepsy was different from that of patients without epilepsy. In order to control for the effect of age on the WB scores, a 4 level factor called AGE was created to classify patients into the age ranges $0-20,20-40,40-60$ and 60+. To determine whether AGE or epilepsy affected the pattern of responses on the survey a repeated measures ANOVA with the response to each question as the repeated measure and AGE and epilepsy as the independent factors.

In order to take a global view whether AED usage had an effect on $\mathrm{WB}$, the patients with true epilepsy were divided into three groups as to whether they were taking no AED, a single AED or polytherapy. The WB scores in these three groups were compared using an ANOVA. A one way ANOVA was used to examine the effect that individual AED's

Tell Us about the Quality of Your Life

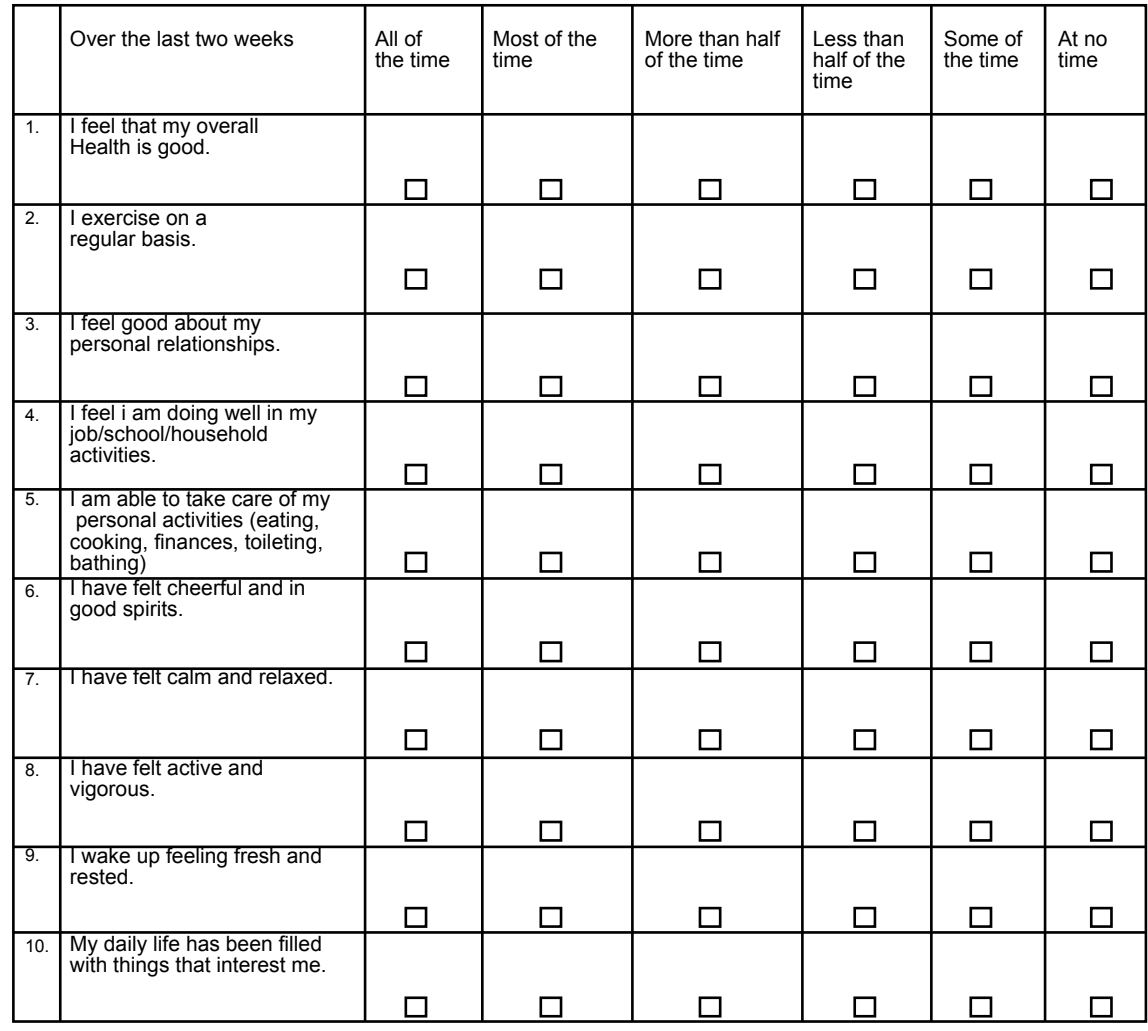

Figure 1: The well being questionnaire used in this study. 
had on WB scores in the group of subjects who had the diagnosis of epilepsy and were on monotherapy.

Spearman rank correlation was used to examine the effect of serial visits on WB scores and in patients with and without epilepsy. Differences in the effect of serial visits in patients with and without epilepsy were confirmed using multiple regression analysis with WB as the dependent variable, and visit number and visit number epilepsy as independent variables. Finding a statistically significant relation between this last variable and the WB indicates a statistically different effect of serial visits on WB in the two groups.

\section{Results}

Significant correlation was found between the well being questions contained in the WHO-5 [7] (questions 6-10) and the new WB questions (questions 1-5) formulated by the investigators (Spearman $\mathrm{R}=0.84, p<.0001$ ). Linear regression analysis showed a strong linear relationship between the total values in the two sets of questions $\left(\mathrm{r}^{2}=\right.$ $0.71, p<.00001)$ with a slope of $0.92(\mathrm{SD}=0.02)$.

Patients with a diagnosis of epilepsy (mean score $=27.6, \mathrm{SD}=$ 14.5), had significantly higher WB scores than those without the formal diagnosis ( mean $=25.2, \mathrm{SD}=12.4), t(756)=212, p<.001$. However, the patients with epilepsy were significantly younger than the other patients in this study (mean age of epileptics $38 \mathrm{SD}=15$, mean age of other patients $49 \mathrm{SD}=17 \mathrm{t}=-6.3 \mathrm{df}=583 \mathrm{p}<.0001)$. In order to determine whether there was a specific effect of epilepsy on the total WB score, factorial ANOVA was performed with the two independent factors of epilepsy and AGE. This demonstrated significant main effects of both epilepsy $(\mathrm{F}(1,524)=6.2, \mathrm{p}<.02)$ and $\operatorname{AGE}(\mathrm{F}(3,524)=3.9, \mathrm{p}<$ $.01)$ as well as a significant interaction $(\mathrm{F}(3,524)=3.5, \mathrm{p}<.02)$.

In order to determine whether there was unique useful information in specific questions, a repeated measure ANOVA was used with epilepsy and AGE as between subject's variables and the scores of each question as a 10 level repeated measures variable. This analysis demonstrated significant main effects of the question number $(\mathrm{F}(9,4716)=78, \mathrm{p}<.001)$. Significant interactions were seen between the question number and epilepsy $(\mathrm{F}(9,4716)=2.8, \mathrm{p}<.005)$ but not between the question number and age $(\mathrm{F}(27,4716)=1.2, \mathrm{p}=.14)$. No significant 3 way interaction between AGE, epilepsy, and question number $(\mathrm{F}(27,4716)=1, \mathrm{p}=.5)$ is seen. Figure 2 shows that patients with epilepsy had lowest WB scores on question 5 compared to patients without epilepsy while the WB scores were most similar on questions 8 and 9.

Analysis of variance showed that among the patients with epilepsy, being on no therapy, monotherapy, or polytherapy had no significant effect on WB $(F(2,215)=.48, p=.6)$. Similar results were obtained when the analysis was repeated using only the WHO-5 questions [7]. ANOVA also showed that there was no effect of anticonvulsant choice on the well being $(F(10,102)=.8256, p=.6)$ in patients treated with AED's in monotherapy. Again, similar results were yielded when analysis was performed using only the WHO-5 questions [7].

Spearman rank correlation demonstrated a significant $\mathrm{R}=.19$ ( $\mathrm{p}<$ .001 ) increase in WB with successive visits in patients with epilepsy but a non significant $\mathrm{R}=-0.05$ ( $\mathrm{p}>.05$ ) in patients without epilepsy. Similar results were found when the used only the WHO-5 questions [7] similar results were found demonstrating a significant $\mathrm{R}=.22$ ( $\mathrm{p}<$ $.001)$ increase in WB with successive visits in patients with epilepsy but a non significant $\mathrm{R}=-0.01$ ( $\mathrm{p}>.05$ ) in patients without epilepsy.

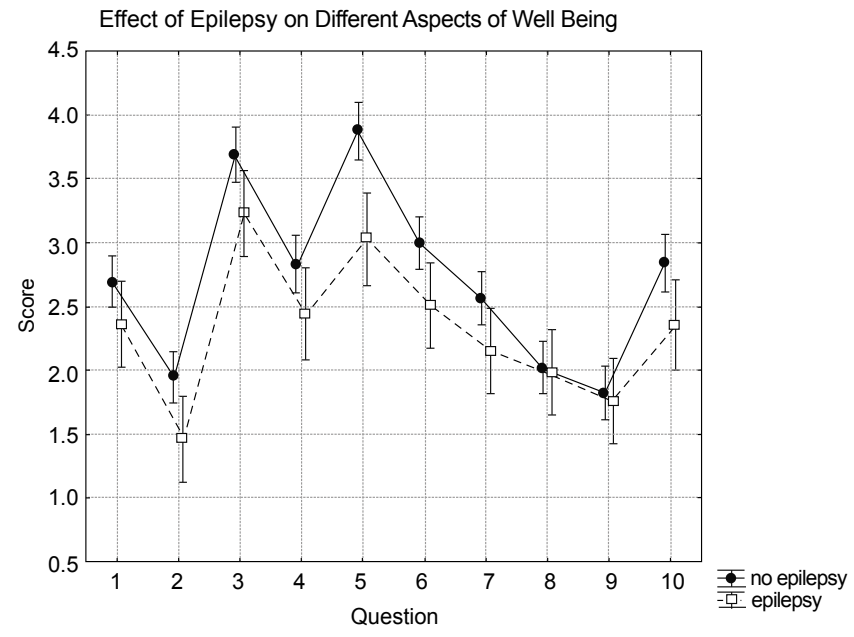

Figure 2: Results of repeated measures ANOVA showing the mean score on each question in the WB survey as a function of the variable epilepsy.

\section{Discussion}

The WHO-5 [7] is an extensively validated [8,9] index of well being. The finding in this paper of a very strong correlation between this index of WB and the other 5 other questions that cover different aspects of WB provides additional support to the validity of this measure in the group of patients studied. The potential value of the simple WHO WB survey is also supported by the fact that the scores from more complex instruments such as the SF-36 [10] and the QOLIE [11] which provide a more detailed picture of the patient's QOL correlate strongly with the scores on the WHO-5 questions $[8,12]$.

Although the WB survey used in this study (Figure 1) is valid measure of WB, the most important result of this pilot study is that this simple questionnaire provides sufficient power to elucidate significant effects in complex populations. Specifically, the survey found that age had a significant effect on WB in the patient population studied. After controlling for this effect, there was a small but significant reduction in WB scores in patients with epilepsy. A repeated measures ANOVA demonstrated that this difference mostly came from answers to all questions except 8 and 9 with the largest difference being in question 5 (Figure 2), a question formulated by the investigators, on the WB questionnaire (Figure 1). This is very much expected in epilepsy patients who may feel vigorous but cannot conduct all of their own activities because epilepsy restricts important functions, such as driving.

Among the patients with diagnosed epilepsy, no significant difference in WB was observed between those on no therapy, monotherapy and polytherapy. Within the monotherapy group AED selection had no statistically significant effect on WB. Other reports however have demonstrated decreases in QOL in patients on polytherapy [13]. In addition, there are more adverse effects in patients on polytherapy [14] and adverse effects have been found to be possibly responsible for decreasing the QOL [15]. Also, other studies have demonstrated differences between AED's in both effectiveness [16] and adverse effects [3,17], both which impact QOL [18]. The difference between these results may be due to three factors. First, many of the studies [15] included patients with pharmacoresistant epilepsy which are more likely to take higher doses of medications and have more side 
effects. Another possibility is that the small number of patients taking AED's in monotherapy reduced this survey's ability to detect such effects. The third possibility is that seizure frequency, which was not reliably quantified in this study, plays a significant role in WB that does not covary with any of the other variables.

Finally, the survey suggested that WB improved over serial visits in patients with epilepsy. This improvement may be due to either treatment of the patient's condition or the establishment of rapport with the provider and education of the patient. When comparing this finding to the literature, both a decrease in seizure frequency and continuity of care, have been found to increase QOL $[18,19]$. It is postulated that this is likely to be related to effective medical treatment since the survey did not indicate a change in WB among the patients who did not have epilepsy who would have been provided with similar support and education. It is also possible that the improvement in $\mathrm{WV}$ in the epilepsy patients may be because the clinic was primarily focused on epilepsy, so the patients with epilepsy received better overall care than patients with other diagnoses. This is consistent with the fact that better care in epilepsy is provided by the most specialized providers [20]. This is an important issue and will be the subject of future investigations.

As clinicians in the neurology clinic strive to improve the QOL of their patients as well as detect the possible comorbidities that have been associated with both neurological disorders [21], and their treatment [22] it is important to take into account the WB of the patient. In order to do this numerous surveys have been formulated to assess QOL. While these studies have proven to be valid, they are fairly long and possibly too complicated for with neurologic problems. One solution is to use a very simple survey such as, the WHO-5 well being index [7] or the extended WB survey used in this paper.

\section{Acknowledgements}

The authors wish to thank Carrie Cox, Brianna McCoy and Hannah Brewer for their help in obtaining the data for this paper.

\section{References}

1. Carson AJ, Ringbauer B, MacKenzie L, Warlow C, Sharpe M (2000) Neurological disease, emotional disorder, and disability: they are related: a study of 300 consecutive new referrals to a neurology outpatient department. J Neurol Neurosurg Psychiatry 68: 202-206.

2. Greenwood RS (2000) Adverse Effects of Antiepileptic Drugs. Epilepsia 41: S42-S52.

3. Kalinin VV (2007) Suicidality and antiepileptic drugs: is there a link? Drug Saf 30: 123-142.

4. Ettinger AB, Devinsky O, Weisbrot DM, Ramakrishna RK, Goyal A (1999) A comprehensive profile of clinical, psychiatric, and psychosocial characteristics of patients with psychogenic nonepileptic seizures. Epilepsia 40: 1292-1298.

5. Oort FJ (2005) Using structural equation modeling to detect response shifts and true change. Qual Life Res 14: 587-598.

6. Diener E (2009) Subjective Well-Being: The Science of Well-Being. Springer, Netherlands.

7. Europe WHOROf. Wellbeing measures in primary health care: the DepCare project: report on a WHO meeting Stockholm, Sweden 12-13 February 1998: WHO Regional Office for Europe; 1998.

8. Bech P, Olsen LR, Kjoller M, Rasmussen NK (2003) Measuring well-being rather than the absence of distress symptoms: a comparison of the SF-36 Mental Health subscale and the WHO-Five well-being scale. Int $\mathrm{J}$ Methods Psychiatr Res 12: 85-91.

9. Bonsignore M, Barkow K, Jessen F, Heun R (2001) Validity of the five-item
WHO Well-Being Index (WHO-5) in an elderly population. Eur Arch Psychiatry Clin Neurosci 251: 1127-1131.

10. Ware JE (1999) SF-36 Health Survey. The use of psychological testing for treatment planning and outcomes assessment. (2ndedn), Mahwah, NJ, US Lawrence Erlbaum Associates Publishers.

11. Cramer JA, Perrine K, Devinsky O, Meador K (1996) A Brief Questionnaire to Screen for Quality of Life in Epilepsy: the QOLIE-10. Epilepsia 37: 577-582.

12. Newnham EA, Hooke GR, Page AC (2010) Monitoring treatment response and outcomes using the World Health Organization's Wellbeing Index in psychiatric care. J Affect Disord 122: 133-138.

13. Thomas SV, Koshy S, Nair CR, Sarma SP (2005) Frequent seizures and polytherapy can impair quality of life in persons with epilepsy. Neurol India 53: 46-50.

14. Fink $P$, Hansen MS, Sondergaard L, Frydenberg M (2003) Mental illness in new neurological patients. J Neurol Neurosurg Psychiatr 74: 817-819.

15. Luoni C, Bisulli F, Canevini MP, De Sarro G, Fattore C, et al. (2011) Determinants of health-related quality of life in pharmacoresistant epilepsy: Results from a large multicenter study of consecutively enrolled patients using validated quantitative assessments. Epilepsia 52: 2181-2191.

16. Arif H, Buchsbaum R, Pierro J, Whalen M, Sims J, et al. (2010) Comparative Effectiveness of 10 Antiepileptic Drugs in Older Adults With Epilepsy. Arch Neurol 67: 408-415.

17. Weintraub D, Buchsbaum R, Resor Jr SR, Hirsch LJ (2007) Psychiatric and behavioral side effects of the newer antiepileptic drugs in adults with epilepsy. Epilepsy Behav 10: 105-110.

18. Baker GA, Jacoby A, Buck D, Stalgis C, Monnet D (1997) Quality of Life of People with Epilepsy: A European Study. Epilepsia 38: 353-362.

19. van Servellen G, Fongwa M, D'errico EM (2006) Continuity of care and quality care outcomes for people experiencing chronic conditions: A literature review. Nurs Health Sci 8: 185-195.

20. Baran M, Stecker MM (2007) Epilepsy in a rural elderly population. Epileptic Disord 9: 256-270.

21. Berlin RM, Ronthal M, Bixler EO, Kales JD (1983) Psychiatric symptomatology in an outpatient neurology clinic. J Clin Psychiatry 44: 204-206.

22. Arana A, Wentworth CE, Ayuso-Mateos JL, Arellano FM (2010) Suicide-related events in patients treated with antiepileptic drugs. N Engl J Med 363: 542-551. 